\title{
SPINOR BUNDLES ON QUADRICS
}

\author{
GIORGIO OTTAVIANI
}

\begin{abstract}
We define some stable vector bundles on the complex quadric hypersurface $Q_{n}$ of dimension $n$ as the natural generalization of the universal bundle and the dual of the quotient bundle on $Q_{4} \simeq \operatorname{Gr}(1,3)$. We call them spinor bundles. When $n=2 k-1$ there is one spinor bundle of rank $2^{k-1}$. When $n=2 k$ there are two spinor bundles of rank $2^{k-1}$. Their behavior is slightly different according as $n \equiv 0(\bmod 4)$ or $n \equiv 2(\bmod 4)$. As an application, we describe some moduli spaces of rank 3 vector bundles on $Q_{5}$ and $Q_{6}$.
\end{abstract}

Introduction. Let $Q_{n}$ be the smooth quadric hypersurface of the complex projective space $\mathbb{P}^{n+1}$.

In this paper we define in a geometrical way some vector bundles on the quadric $Q_{n}$ as the natural generalization of the universal bundle and the dual of the quotient bundle on $Q_{4} \simeq \operatorname{Gr}(1,3)$. We call them spinor bundles. On $Q_{4}$ this definition is equivalent to the usual one.

Spinor bundles are homogeneous and stable (according to the definition of Mumford-Takemoto). We study their first properties using the geometrical description given and some standard techniques available in [OSS].

We also use a theorem of Ramanan (see [Um]) about the stability of homogeneous bundles induced by irreducible representations. When $n$ is odd there is only one spinor bundle, while when $n$ is even there are two nonisomorphic spinor bundles. When $n$ is even the behavior of spinor bundles is slightly different according as $n \equiv 0(\bmod 4)$ or $n \equiv 2(\bmod 4)$.

In [Ot2] we have given a cohomological splitting criterion for vector bundles on quadrics involving spinor bundles.

$Q_{n} \simeq \operatorname{Spin}(n+2) / P\left(\alpha_{1}\right)[\mathbf{S t}]$ is a homogeneous manifold, and the semisimple part of the Lie algebra of $P\left(\alpha_{1}\right)$ is $\mathfrak{o}(n)$. At the level of Lie algebras, spinor bundles are defined from the spin and half-spin representations of $\mathfrak{o}(n)$.

The paper is divided into three sections. In $\S 1$ we give some preliminary results and we define the spinor bundles. In $\S 2$ we study the first properties of spinor bundles. In $\S 3$, as an application, we describe some moduli spaces of rank 3 vector bundles on $Q_{5}$ and $Q_{6}$.

1. Geometrical definition of spinor bundles on $Q_{n}$. For basic facts about vector bundles we refer to $[\mathrm{OSS}]$. We set $E(t)=E \otimes \mathcal{O}_{Q_{n}} O_{Q_{n}}(t)$ for $t \in \mathbb{Z}$ when

Received by the editors March 31, 1987.

1980 Mathematics Subject Classification (1985 Revision). Primary 14F05; Secondary 14M17, $32 \mathrm{M} 10$.

Key words and phrases. Vector bundle, homogeneous, stable, spinor, moduli space.

This paper was written while the author was enrolled in the Research Doctorate of the University of Florence. Partially supported by MPI $40 \%$ funds. 
$E$ is a vector bundle on $Q_{n}$. The first Chern class of $E$ can be considered as an integer. We denote by $E^{*}$ the dual vector bundle of $E$. If $F$ is a sheaf on a variety $X$, we denote by $h^{i}(X, F)$ the dimension of the complex vector space $H^{i}(X, F)$.

We will recall some facts about linear spaces of maximal dimension on $Q_{n}[\mathbf{G H}$, Chapter 6; LV, pp. 16-17].

The situation is different for quadrics of odd or even dimension. If $n=2 k$ is even, then the linear spaces of $Q_{n}$ of maximal dimension have dimension $k$. There are two disjoint families of $k$-planes on $Q_{n}$, each parametrized by a smooth irreducible projective variety $S_{k}$ (called spinor variety) of dimension $k(k+1) / 2$. We have: $\operatorname{Pic}\left(S_{k}\right) \simeq \mathbb{Z}$ and if $O_{S_{k}}(1)$ is the ample generator, then $H^{0}\left(S_{k}, O_{S_{k}}(1)\right)=\mathbb{C}^{2^{k}}$.

If $n=2 k+1$ is odd, then the linear spaces on $Q_{n}$ of maximal dimension have dimension $k$. There is only one family of $k$-planes on $Q_{n}$ which is parametrized by $S_{k+1}$.

It is well known that $S_{1} \simeq \mathbb{P}^{1}, S_{2} \simeq \mathbb{P}^{3}$.

Let $Q_{2 k+2} \cap H=Q_{2 k+1}$ with $H$ a nontangent hyperplane. The natural map

$$
\begin{aligned}
\left\{\mathbb{P}^{k+1} \mid \mathbb{P}^{k+1} \subset Q_{2 k+2}\right\} & \rightarrow\left\{\mathbb{P}^{k} \mid \mathbb{P}^{k} \subset Q_{2 k+1}\right\}, \\
\mathbb{P}^{k+1} & \mapsto \mathbb{P}^{k+1} \cap H
\end{aligned}
$$

is $2: 1$ and is an isomorphism when restricted to each connected component of $\left\{\mathbb{P}^{k+1} \mid \mathbb{P}^{k+1} \subset Q_{2 k+2}\right\}$. Observe that if $\mathbb{P}^{k+1} \subset H$ for some $\mathbb{P}^{k+1}$, then $H$ would be tangent to $Q_{2 k+2}$, so that (1) is well defined.

If $x \in Q_{n}$, the linear spaces on $Q_{n}$ of maximal dimension which contain $x$ are in a natural bijective correspondence with the linear spaces of maximal dimension on $Q_{n-2} \simeq Q_{n} \cap T_{x} Q_{n} \cap H$, where $T_{x} Q_{n}$ is the tangent space to $Q_{n}$ in $x$ and $H$ is a generic hyperplane. In fact $T_{x} Q_{n} \cap Q_{n}$ is a cone with vertex in $x$ over a smooth quadric $Q_{n-2}$.

PROPOSITION 1.1. Let $Q_{2 k+1} \cap H=Q_{2 k}$, where $H$ is a nontangent hyperplane. We have $\left\{\mathbb{P}^{k} \mid \mathbb{P}^{k} \subset Q_{2 k}\right\} \simeq S_{k}^{\prime} \cup S_{k}^{\prime \prime}$, so that we have two natural embeddings $i^{\prime}: S_{k}^{\prime} \rightarrow S_{k+1}$ and $i^{\prime \prime}: S_{k}^{\prime \prime} \rightarrow S_{k+1}$, where $S_{k+1} \simeq\left\{\mathbb{P}^{k} \mid \mathbb{P}^{k} \subset Q_{2 k+1}\right\}$.

Then $i^{\prime *} O_{S_{k+1}}(1) \simeq O_{S_{k}^{\prime}}(1), i^{\prime \prime *} O_{S_{k+1}}(1) \simeq O_{S_{k}^{\prime \prime}}(1)$, and the restriction map

$$
H^{0}\left(S_{k+1}, O(1)\right) \rightarrow H^{0}\left(S_{k}^{\prime}, O(1)\right) \oplus H^{0}\left(S_{k}^{\prime \prime}, O(1)\right)
$$

is an isomorphism

PROOF. We have a natural commutative diagram

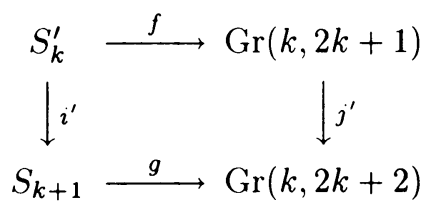

where $j^{*} O_{\mathrm{Gr}(k, 2 k+2)}(1) \simeq O_{\mathrm{Gr}(k, 2 k+1)}(1)$.

It is shown in $[\mathbf{L V}]$ that $f^{*} O_{\mathrm{Gr}(k, 2 k+2)}(1) \simeq \mathcal{O}_{S_{k+1}}(2)$. Then

$$
i^{\prime *} O_{S_{k+1}}(2)=\left(g \circ i^{\prime}\right)^{*} O_{\mathrm{Gr}(k, 2 k+2)}(1)=(j \circ f)^{*} O_{\mathrm{Gr}(k, 2 k+2)}(1) \simeq O_{S_{k}^{\prime}}(2) \text {. }
$$

$\operatorname{As} \operatorname{Pic}\left(S_{k}^{\prime}\right)=\mathbb{Z}$, it follows that $i^{\prime *} O_{S_{k+1}}(1) \simeq O_{S_{k}^{\prime}}(1)$ and in the same way $i^{\prime \prime *} O_{S_{k+1}}(1)$ $\simeq O_{S_{k}^{\prime \prime}}(1)$. 
For the second assertion, since $H^{0}\left(S_{k+1}, O(1)\right)$ and $H^{0}\left(S_{k}^{\prime}, O(1)\right) \oplus H^{0}\left(S_{k}^{\prime \prime}, O(1)\right)$ are vector spaces of the same dimension $2^{k+1}$, it is sufficient to prove that the restriction map is injective.

We use induction on $k$. If $k=1$, then $i^{\prime}\left(S_{1}^{\prime}\right)$ and $i^{\prime \prime}\left(S_{1}^{\prime \prime}\right)$ are two skew lines in $\mathbb{P}^{3}$, so that the assertion is true.

Suppose that the assertion is false for $k+1$ : we have $s \in H^{0}\left(S_{k+2}, O(1)\right), s \neq 0$, such that $\left.s\right|_{S_{k+1}^{\prime}} \equiv 0,\left.s\right|_{S_{k+1}^{\prime \prime}} \equiv 0$. Let $\tilde{\mathbb{P}}^{k+1} \subset Q_{2 k+3}$ such that $s\left(\tilde{\mathbb{P}}^{k+1}\right) \neq 0$ and let $x \in \tilde{\mathbf{P}}^{k+1} \cap H$. Then

$$
\left\{\mathbb{P}^{k+1} \mid x \in \mathbb{P}^{k+1} \subset Q_{2 k+3} \cap H\right\} \quad \hookrightarrow \quad\left\{\mathbb{P}^{k+1} \mid x \in \mathbb{P}^{k+1} \subset Q_{2 k+3}\right\}
$$

and we obtain the natural commutative diagram:

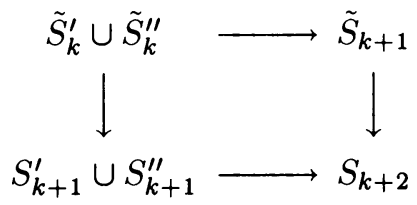

We have $\left.s\right|_{\tilde{S}_{k+1}} \neq 0$ because $\tilde{\mathbb{P}}^{k+1} \in \tilde{S}_{k+1}$, but $\left.\left.s\right|_{\tilde{S}_{k}^{\prime}} \equiv s\right|_{\tilde{S}_{k}^{\prime \prime}} \equiv 0$, contradicting the induction hypothesis. This completes the proof.

COROLlARY 1.2. (i) Let $x \in Q_{2 k+1}$ and consider

$$
\left(S_{k}\right)_{x}:=\left\{\mathbb{P}^{k} \mid x \in \mathbb{P}^{k} \subset Q_{2 k+1}\right\} \stackrel{i_{x}}{\hookrightarrow}\left\{\mathbb{P}^{k} \mid \mathbb{P}^{k} \subset Q_{2 k+1}\right\}=S_{k+1} .
$$

Then $i_{x}^{*} O_{S_{k+1}}(1) \simeq O_{\left(S_{k}\right)_{x}}(1)$ and the restriction map

$$
H^{0}\left(S_{k+1}, O(1)\right) \rightarrow H^{0}\left(\left(S_{k}\right)_{x}, O(1)\right)
$$

is surjective.

(ii) Let $x \in Q_{2 k}$ and consider

$$
\left(S_{k-1}^{\prime}\right)_{x} \cup\left(S_{k-1}^{\prime \prime}\right)_{x}=\left\{\mathbb{P}^{k} \mid x \in \mathbb{P}^{k} \subset Q_{2 k}\right\} \stackrel{j_{x}}{\hookrightarrow}\left\{\mathbb{P}^{k} \mid \mathbb{P}^{k} \subset Q_{2 k}\right\}=S_{k}^{\prime} \cup S_{k}^{\prime \prime} .
$$

$j_{x}$ induces two embeddings:

$$
j_{x}^{\prime}:\left(S_{k-1}^{\prime}\right)_{x} \rightarrow S_{k}^{\prime}, \quad j_{x}^{\prime \prime}:\left(S_{k-1}^{\prime \prime}\right)_{x} \rightarrow S_{k}^{\prime \prime} .
$$

Then $j_{x}^{\prime *} O_{S_{k}^{\prime}}(1) \simeq O_{\left(S_{k-1}^{\prime}\right)}(1), j_{x}^{\prime \prime *} O_{S_{k}^{\prime \prime}}(1) \simeq O_{\left(S_{k-1}^{\prime \prime}\right)_{x}}(1)$ and the restriction maps

$$
\begin{aligned}
& H^{0}\left(S_{k}^{\prime}, O(1)\right) \rightarrow H^{0}\left(\left(S_{k-1}^{\prime}\right)_{x}, O(1)\right), \\
& H^{0}\left(S_{k}^{\prime \prime}, O(1)\right) \rightarrow H^{0}\left(\left(S_{k-1}^{\prime \prime}\right)_{x}, O(1)\right)
\end{aligned}
$$

are surjective.

Proof. We have observed that $\left\{\mathbb{P}^{k} \mid x \in \mathbb{P}^{k} \subset Q_{2 k+1}\right\} \simeq\left\{\mathbb{P}^{k-1} \mid \mathbb{P}^{k-1} \subset\right.$ $\left.Q_{2 k+1} \cap T_{x} Q_{2 k+1} \cap H\right\}$ where $H$ is a hyperplane not tangent to $Q_{2 k+1}$ and not containing $x$.

From map (1) we see that the last variety is isomorphic to one component of $\left\{\mathbf{P}^{k} \mid \mathbf{P}^{k} \subset Q_{2 k+1} \cap H\right\}$. Now the thesis of case (i) follows from Proposition 1. 
In case (ii), we cut with a hyperplane $H$, so that from map (1) we go back again to case (i).

Consider now the quadric $Q_{2 k+1}$. From Corollary 1.2, for each $x \in Q_{2 k+1}$ we have an inclusion

$$
H^{0}\left(\left(S_{k}\right)_{x}, O(1)\right)^{*} \rightarrow H^{0}\left(S_{k+1}, O(1)\right)^{*}
$$

and then an embedding $s: Q_{2 k+1} \rightarrow \operatorname{Gr}\left(2^{k}-1,2^{k+1}-1\right)$ in the Grassmannian of $\left(2^{k}-1\right)$-subspaces of $\mathbb{P}^{2^{k+1}-1}$.

In the same way, in the case of even dimension, from Corollary 1.2 we have the two embeddings

$$
s^{\prime}: Q_{2 k} \rightarrow \operatorname{Gr}\left(2^{k-1}-1,2^{k}-1\right), \quad s^{\prime \prime}: Q_{2 k} \rightarrow \operatorname{Gr}\left(2^{k-1}-1,2^{k}-1\right) .
$$

DEFINITION 1.3. Let $U$ be the universal bundle of the Grassmannian. We call $s^{*} U=S$ the spinor bundle on $Q_{2 k+1}$. Its rank is $2^{k}$. We call $s^{*} U=S^{\prime}$ and $s^{\prime \prime *} U \simeq S^{\prime \prime}$ the two spinor bundles on $Q_{2 k}$. Their rank is $2^{k-1}$.

If $f$ is an automorphism of $Q_{2 k}$ that exchanges the two families of $k$-planes, we have $f^{*} S^{\prime} \simeq S^{\prime \prime}$ and $f^{*} S^{\prime \prime} \simeq S^{\prime}$.

It is clear that spinor bundles $S$ on all quadrics $Q$ are homogeneous, i.e., $f^{*} S \simeq$ $S \forall f \in \operatorname{Aut}(Q)^{0}$, where $\operatorname{Aut}(Q)^{0}$ is the connected component of the identity in $\operatorname{Aut}(Q)$.

From the geometrical description given, the following theorem is clear.

THEOREM 1.4. (i) Let $S^{\prime}, S^{\prime \prime}$ be the spinor bundles on $Q_{2 k}$, and let $i: Q_{2 k-1} \rightarrow$ $Q_{2 k}$ be a smooth hyperplane section. Then $i^{*} S^{\prime} \simeq i^{*} S^{\prime \prime} \simeq S$, where $S$ is the spinor bundle on $Q_{2 k-1}$.

(ii) Let $S$ be the spinor bundle on $Q_{2 k+1}$, and let $i: Q_{2 k} \rightarrow Q_{2 k+1}$ be a smooth hyperplane section. Then $i^{*} S \simeq S^{\prime} \oplus S^{\prime \prime}$, where $S^{\prime}$ and $S^{\prime \prime}$ are the spinor bundles on $Q_{2 k}$.

EXAMPLES 1.5. In the definition of spinor bundles, the two embeddings $s^{\prime}: Q_{4} \rightarrow \operatorname{Gr}(1,3)$ and $s^{\prime \prime}: Q_{4} \rightarrow \operatorname{Gr}(1,3)$ are isomorphisms. So the spinor bundles on $Q_{4}$ are the universal bundle and the dual of the quotient bundle.

The embedding $s: Q_{3} \rightarrow \operatorname{Gr}(1,3)$ corresponds to a hyperplane section. If $S$ is the spinor bundle on $Q_{3}$, then $S^{2} S^{*}=T Q_{3}$. In fact $\left.T Q_{4}\right|_{Q_{3}} \simeq S^{*} \otimes S^{*} \simeq S^{2} S^{*} \oplus O(1)$ and the exact sequence

$$
\left.0 \rightarrow T Q_{3} \rightarrow T Q_{4}\right|_{Q_{3}} \rightarrow O(1) \rightarrow 0
$$

splits. On $Q_{2}$ the two spinor bundles are the duals of the two line bundles corresponding to two skew-lines on $Q_{2}$.

On $Q_{1} \simeq \mathbb{P}^{1}$ we can define the spinor bundle to be $O_{\mathbb{P}^{1}}(-1)$.

COROLLARY 1.6. Let $l \subset Q_{n}(n \geq 3)$ be a line and let $S$ be a spinor bundle on $Q_{n}$. Then $\left.S\right|_{l}=O_{l}^{\oplus 2^{[(n-3) / 2]}} \oplus O_{l}(-1)^{\oplus 2^{[(n-3) / 2]}}$.

2. First properties of spinor bundles on $Q_{n}$. Now let $n=2 k+1$ and consider the connected, simply connected, simple Lie group $\operatorname{Spin}(n+2)=G_{n}$. We fix a maximal torus $T \subset G_{n}$. The Lie algebra of $G_{n}$ is $\mathfrak{o}(n+2)$ and the Lie algebra of $T$ is a Cartan subalgebra of $\mathfrak{o}(n+2)$ which we denote by $\mathfrak{h}$.

Let $\Phi$ be the set of roots of $\mathfrak{o}(n+2)$ relative to $\mathfrak{h}$, and let $\alpha_{1}, \ldots, \alpha_{k+1}$ be a subset of simple roots, so that every root $\alpha \in \Phi$ can be written as an integral combination 
$\alpha=\sum_{i=1}^{k+1} n_{i} \alpha_{i}$ with either all $n_{i} \geq 0$ or all $n_{i} \leq 0$. This divides the set of roots into two disjoint subsets, the positive roots $\Phi^{+}$, and the negative roots $\Phi^{-}$.

Let $($,$) denote the natural inner product on \mathfrak{h}^{*}$ induced by the Killing form. Let $\lambda_{1}, \ldots, \lambda_{k+1} \in \mathfrak{h}^{*}$ be the fundamental weights, characterized by the property $2\left(\lambda_{i}, \alpha_{j}\right) /\left(\alpha_{j}, \alpha_{j}\right)=\delta_{i j}$. We also denote by $\lambda_{i}$ the corresponding fundamental weights which act on $T$.

Associated to the set of simple roots $\alpha_{1}, \ldots, \alpha_{k+1}$ is the Dynkin diagram $B_{k+1}$. Let $g^{\alpha} \subset \mathfrak{o}(n+2)$ be the eigenspace for a root $\alpha$. If $A \subset\left\{\alpha_{1}, \ldots, \alpha_{k+1}\right\}$ we define $\Phi^{+}(A)=\left\{\alpha \in \Phi^{+} \mid \alpha=\sum_{i=1}^{k+1} n_{i} \alpha_{i}, n_{i}=0\right.$ for $\left.\alpha_{i} \in A\right\}$. Let $P(A)$ be the standard parabolic subgroup of $G_{n}$ whose Lie algebra is $\mathfrak{h} \oplus\left(\bigoplus_{\alpha \in \Phi^{-}} g^{\alpha}\right) \oplus\left(\bigoplus_{\alpha \in \Phi^{+}(A)} g^{\alpha}\right)$. Consider the incidence variety $F:=\left\{\left(x, \mathbb{P}^{k}\right) \in Q_{n} \times S_{k+1} \mid x \in \mathbb{P}^{k} \subset Q_{n}\right\}$.

We have two surjective projections as follows:

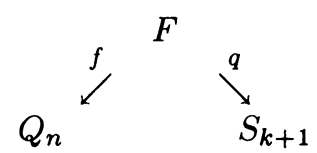

Then, if $x \in Q_{n}, p^{-1}(x) \simeq\left(S_{k}\right)_{x}$ and $\left.q\right|_{p^{-1}(x)}=i_{x}$ (see Corollary 1.2). From the geometrical description given, the dual of the spinor bundle $S^{*}$ on $Q_{n}$ is $p_{*} q^{*} O(1)$, where $O(1)$ is the ample line bundle on $S_{k+1}$ which generates $\operatorname{Pic}\left(S_{k+1}\right)=\mathbb{Z}$. It is well known [St] that in the diagram (2) we have the isomorphisms $Q_{n} \simeq G_{n} / P\left(\alpha_{1}\right)$, $S_{k+1} \simeq G_{n} / P\left(\alpha_{k+1}\right), F \simeq G_{n} / P\left(\alpha_{1}, \alpha_{k+1}\right)$.

The line bundle $O(1)$ on $S_{k+1}$ is defined by the character $\lambda_{k+1}$ of (the semisimple part of) $P\left(\alpha_{k+1}\right)$, that is $O(1)=G_{n} \times_{P\left(\alpha_{k+1}\right)} \mathbb{C}$ where the twisted product is defined to be the quotient of $G_{n} \times \mathbb{C}$ by the action $p \cdot(g, v)=\left(g p^{-1}, \lambda_{k+1}(p) v\right)$, $(g, v) \in G_{n} \times \mathbb{C}, p \in P\left(\alpha_{k+1}\right)$.

Then $q^{*} O(1)$ is a line bundle on $F$ defined by the character $\lambda_{k+1}$ of the semisimple part of $P\left(\alpha_{1}\right)$, and $S^{*}=p_{*} q^{*} O(1)$ is defined by the irreducible representation $\rho$ of the semisimple part of $P\left(\alpha_{1}\right)$ with maximal weight $\lambda_{k+1}$. At the level of Lie algebras, the semisimple part of Lie $P\left(\alpha_{1}\right)$ is $\mathfrak{o}(n)$ (the Dynkin diagram is $B_{k}$ ) and it follows that $D \rho$ is the spin representation of $\mathfrak{o}(n)$.

Exactly in the same way, when $n=2 k, S^{\prime *}$ and $S^{\prime *}$ are defined from the two half-spin representations of $o(n)$.

The important fact is that for each $n$ the spinor bundles are defined by irreducible representations. By a theorem of Ramanan (see [Um]), vector bundles defined by irreducible representations are stable with respect to each ample line bundle, so that we can state the following.

THEOREM 2.1. The spinor bundles on $Q_{n}$ are stable.

We shall use also the now classical theorem of Bott (see [St]) about cohomology of homogeneous bundles.

For further reference, we also state the following.

COROLLARY 2.2. Let $E$ be a vector bundle on $Q_{2 k+1}$ and let $Q_{2 k} \subset Q_{2 k+1}$ be a generic hyperplane section. If $\left.E\right|_{Q_{2 k}} \simeq S^{\prime} \oplus S^{\prime \prime}$ then $E$ is semistable.

THEOREM 2.3. Let $S$ be a spinor bundle on $Q_{n}$. Then

$$
\begin{array}{ll}
H^{i}\left(Q_{n}, S(t)\right)=0 & \text { for } i \text { such that } 0<i<n, \text { for all } t \in \mathbb{Z}, \\
H^{0}\left(Q_{n}, S(t)\right)=0 & \text { for } t \leq 0, h^{0}\left(Q_{n}, S(1)\right)=2^{[(n+1) / 2]} .
\end{array}
$$


PROOF. We could apply the theorem of Bott, but we prefer to give a simple inductive argument.

For $n=1,2$ the theorem is well known. So we proceed by induction on $n$. Let $S$ be a spinor bundle on $Q_{n+1}$. Consider the exact sequences on $Q_{n+1}$ :

$$
\left.0 \rightarrow S(t-1) \rightarrow S(t) \rightarrow S\right|_{Q_{n}}(t) \rightarrow 0 \text { for all } t \in \mathbb{Z}
$$

From the induction hypothesis and Theorem 1.4 we have

$$
\begin{array}{ll}
H^{i}\left(Q_{n},\left.S\right|_{Q_{n}}(t)\right)=0 & \text { for } i \text { such that } 0<i<n, \text { for all } t \in \mathbb{Z}, \\
H^{0}\left(Q_{n},\left.S\right|_{Q_{n}}(t)\right)=0 & \text { for } t \leq 0 .
\end{array}
$$

Then

$$
\begin{array}{ll}
H^{i}\left(Q_{n+1}, S(t)\right)=0 \text { for } i \text { such that } 1<i<n, \text { for all } t \in \mathbb{Z}, \\
H^{1}\left(Q_{n+1}, S(t-1)\right) \rightarrow H^{1}\left(Q_{n+1}, S(t)\right) & \text { is surjective for all } t \in \mathbb{Z}, \\
H^{n}\left(Q_{n+1}, S(t-1)\right) \rightarrow H^{n}\left(Q_{n+1}, S(t)\right) & \text { is injective for all } t \in \mathbb{Z}, \\
H^{0}(S(t-1)) \rightarrow H^{0}(S(t)) & \text { is surjective for } t \leq 0 .
\end{array}
$$

From Theorem B and Serre duality the result follows.

We now want to study the case when spinor bundles restrict to maximal linear spaces on quadrics.

We first need the following lemma.

LEMMA 2.4. Let $E$ be a vector bundle on $\mathbb{P}^{n}$ such that

$$
H^{i}\left(\mathbb{P}^{n}, E(-j)\right)= \begin{cases}\mathbb{C} & \text { if } 0 \leq i=j \leq n, \\ 0 & \text { if } 0 \leq i, j \leq n, i \neq j .\end{cases}
$$

Then

$$
E \simeq \bigoplus_{i=0}^{n} \Omega_{\mathbf{p}^{n}}^{i}(i)
$$

ProOF. We have a Beilinson spectral sequence [Be, OSS] with $E_{1}$-term

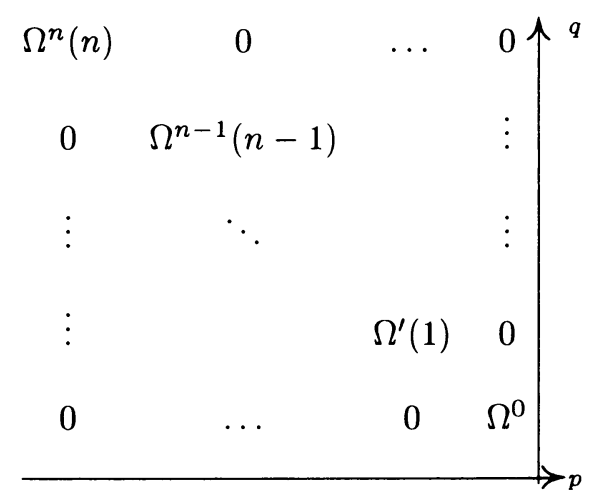

which converges to

$$
E^{i}= \begin{cases}E & \text { for } i=0 \\ 0 & \text { otherwise }\end{cases}
$$


In our case $E_{1}^{p, q}=E_{2}^{p, q}=E_{\infty}^{p, q}$, so that a filtration of $E$ results: $0=E_{0} \subset E_{1} \subset$ $\cdots \subset E_{n}=E$ with $E_{i}$ subsheaves such that

$$
\bigoplus_{i=0}^{n} E_{i} / E_{i-1} \simeq \bigoplus_{p=0}^{n} E_{\infty}^{-p, p} \simeq \bigoplus_{i=0}^{n} \Omega^{i}(i) .
$$

Consider the sequence

$$
0 \rightarrow E_{1} \rightarrow E_{2} \rightarrow E_{2} / E_{1} \rightarrow 0 \text {. }
$$

By [At], we have $E_{1} \simeq \Omega^{p}(p), E_{2} / E_{1} \simeq \Omega^{q}(q)$ for some $p, q$. By Lemma 2 of $[\mathbf{B e}]: \operatorname{Ext}^{1}\left(\Omega^{q}(q), \Omega^{p}(p)\right)=0$, so that $E_{2} \simeq \Omega^{p}(p) \oplus \Omega^{q}(q)$.

Exactly in the same way it follows that each subsheaf $E_{i}$ is a direct summand of $E_{i+1}$, and this completes the proof.

THEOREM 2.5. Let $S$ be a spinor bundle on $Q_{2 m+1}$ and consider a linear space $\mathbb{P}^{m} \subset Q_{2 m+1}$. Then $\left.S\right|_{\mathbf{p}^{m}}=\bigoplus_{i=0}^{m} \Omega_{\mathbf{p}_{m}}^{i}(i)$.

PROOF. The proof is by induction on $m$. For $m=1$ the result is well known. By Lemma 2.4 it is sufficient to show that

$$
H^{i}\left(\mathbb{P}^{m},\left.S\right|_{\mathbf{P} m}(-j)\right)= \begin{cases}\mathbb{C} & \text { for } 0 \leq i=j \leq m, \\ 0 & \text { for } 0 \leq i, j \leq m, i \neq j .\end{cases}
$$

Consider a linear space $\mathbb{P}^{m+1} \supset \mathbb{P}^{m}$. Then $\mathbb{P}^{m+1} \cap Q_{2 m+1}=\mathbb{P}^{m} \cup \tilde{\mathbb{P}}^{m}:=X$ and we have an exact sequence of Mayer-Vietoris type [Ba]

$$
0 \rightarrow O_{X} \rightarrow O_{\mathbf{P} m} \oplus O_{\tilde{\mathbf{P}}^{m}} \rightarrow O_{Y} \rightarrow 0
$$

where $Y=\mathbb{P}^{m} \cap \tilde{\mathbb{P}}^{m} \simeq \mathbb{P}^{m-1}$.

Consider a generic linear space $\mathbb{P}^{2 m} \subset \mathbb{P}^{2 m+2}$ such that $\mathbb{P}^{2 m} \supset Y$. Then $\mathbb{P}^{m} \cap \mathbb{P}^{2 m}=Y, \mathbb{P}^{2 m} \cap Q_{2 m+1}=Q_{2 m-1}$ smooth quadric.

We get the commutative diagram:

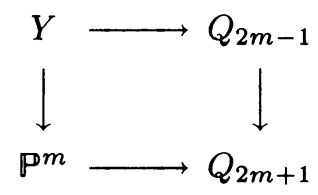

Thus by Theorem 1.4 $\left.\left.\left.S\right|_{Y} \simeq S_{Q_{2 m-1}}\right|_{Y} \oplus S_{Q_{2 m-1}}\right|_{Y}$, where $S_{Q_{2 m-1}}$ is the spinor bundle on $Q_{2 m-1}$. By the induction hypothesis, $\left.S_{Q_{2 m-1}}\right|_{Y} \simeq \bigoplus_{i=0}^{m-1} \Omega_{Y}^{i}(i)$. Then

$$
\begin{gathered}
H^{i}\left(Y,\left.S\right|_{Y}(-j)\right)= \begin{cases}\mathbb{C}^{2}, & 0 \leq i=j \leq m-1, \\
0, & 0 \leq i, j \leq m-1, i \neq j,\end{cases} \\
h^{m-1}\left(Y,\left.S\right|_{Y}(-m)\right)=2 \sum_{i=0}^{m-1}\left(\begin{array}{c}
m \\
i
\end{array}\right)=2^{m+2}-2 .
\end{gathered}
$$

Moreover, as $X$ is a (singular) plane section of $Q_{2 m+1}$, it is easy to check inductively that

$$
\begin{gathered}
H^{0}\left(X,\left.S\right|_{X}(-j)\right)=0 \text { for } j \geq 0 \\
H^{i}\left(X,\left.S\right|_{X}(-j)\right)=0 \text { for } 1 \leq i \leq m-1, \forall j \in \mathbb{Z}
\end{gathered}
$$




$$
h^{m}\left(X,\left.S\right|_{X}(-j)\right)= \begin{cases}0 & \text { for } j \leq m-1 \\ 2^{m+1} & \text { for } j=m .\end{cases}
$$

If we tensor (3) by $S$ and consider the associated cohomology sequence we get

$$
H^{i}\left(\mathbf{P}^{m},\left.S\right|_{\mathbf{P}^{m}}(-j)\right) \oplus H^{i}\left(\tilde{\mathbf{P}}^{m},\left.S\right|_{\tilde{\mathbf{P}} m}(-j)\right)= \begin{cases}\mathbb{C}^{2} & \text { for } 0 \leq i=j \leq m, \\ 0 & \text { for } 0 \leq i, j \leq m, i \neq j .\end{cases}
$$

As $S$ is homogeneous, we have $\left.\left.S\right|_{\mathbf{p} m} \simeq S\right|_{\tilde{\mathbf{p}} m}$. This completes the proof.

THEOREM 2.6. (i) Let $S^{\prime}, S^{\prime \prime}$ be the spinor bundles on $Q_{4 m}$. The restrictions to one family of linear $\mathbf{P}^{2 m} \subset Q_{4 m}$ are

$$
\left.S^{\prime}\right|_{\mathbf{p}^{2 m}} \simeq \bigoplus_{i=0}^{m} \Omega_{\mathbf{P}^{2 m}}^{2 i}(2 i),\left.\quad S^{\prime \prime}\right|_{\mathbf{p}^{2 m}} \simeq \bigoplus_{i=0}^{m-1} \Omega_{\mathbf{P}^{2 m}}^{2 i+1}(2 i+1),
$$

while the restrictions to the other family of linear $\mathbb{P}^{2 m} \subset Q_{4 m}$ are

$$
\left.S^{\prime}\right|_{\mathbf{p}^{2 m}} \simeq \bigoplus_{i=0}^{m-1} \Omega_{\mathbf{P}^{2 m}}^{2 i+1}(2 i+1),\left.\quad S^{\prime \prime}\right|_{\mathbf{p}^{2 m}} \simeq \bigoplus_{i=0}^{m} \Omega_{\mathbf{p}^{2 m}}^{2 i}(2 i) .
$$

(ii) Let $S^{\prime}, S^{\prime \prime}$ be the spinor bundles on $Q_{4 m+2}$. The restrictions to one family of linear $\mathbf{P}^{2 m+1} \subset Q_{4 m+2}$ are

$$
\left.S^{\prime}\right|_{\mathbf{P}^{2 m+1}} \simeq \bigoplus_{i=0}^{m} \Omega_{\mathbf{P}^{2 m+1}}^{2 i}(2 i),\left.\quad S^{\prime \prime}\right|_{\mathbf{P}^{2 m+1}} \simeq \bigoplus_{i=0}^{m} \Omega_{\mathbf{P}^{2 m+1}}^{2 i+1}(2 i+1),
$$

while the restrictions to the other family of linear $\mathbf{P}^{2 m+1} \subset Q_{4 m+2}$ are

$$
\left.S^{\prime}\right|_{\mathbf{p}^{2 m+1}} \simeq \bigoplus_{i=0}^{m} \Omega_{\mathbf{P}^{2 m+1}}^{2 i+1}(2 i+1),\left.\quad S^{\prime \prime}\right|_{\mathbf{p}^{2 m+1}} \simeq \bigoplus_{i=0}^{m} \Omega_{\mathbf{P}^{2 m+1}}^{2 i}(2 i) .
$$

ProOF. Let $\mathbf{P}^{k} \subset Q_{2 k}$ be a linear space. From Theorems 1.4 and 2.5 it follows that $\left.S^{\prime} \oplus S^{\prime \prime}\right|_{\mathbf{p} k} \simeq \bigoplus_{i=0}^{k} \Omega_{\mathbf{p} k}^{i}(i)$. By [At] $S^{\prime}$ and $S^{\prime \prime}$ must decompose on $\mathbf{P}^{k}$ with direct summands $\Omega_{\mathbf{p}^{k}}^{i}(i)$ for some $i$.

Consider now a hyperplane $\mathbb{P}^{k-1} \subset \mathbb{P}^{k}$. As $\left.\Omega_{\mathbf{p}^{k}}^{1}(1)\right|_{\mathbf{p}^{k-1}} \simeq \Omega_{\mathbf{p}^{k-1}}^{1}(1) \oplus \mathcal{O}_{\mathbf{p}^{k-1}}$, from the canonical isomorphism $\bigwedge^{n}(A \oplus B)=\bigoplus_{j=0}^{n}\left(\bigwedge^{j} A \otimes \bigwedge^{n-j} B\right)(A, B$ vector bundles) we get

$$
\left.\Omega_{\mathbf{P}^{k}}^{i}(i)\right|_{\mathbf{P}^{k-1}} \simeq \Omega_{\mathbf{P}^{k-1}}^{i}(i) \oplus \Omega_{\mathbf{P}^{k-1}}^{i-1}(i-1) .
$$

By Theorem 1.4, $\left.\left.S^{\prime}\right|_{\mathbf{P}^{k-1}} \simeq S^{\prime \prime}\right|_{\mathbf{P}^{k-1}}$, so that it follows that the only possible decompositions are those stated.

LEMMA 2.7. Let $E_{1}, E_{2}$ be semistable vector bundles on $Q_{n}$ with $\operatorname{rank} E_{1}=$ $\operatorname{rank} E_{2}, c_{1}\left(E_{1}\right)=c_{1}\left(E_{2}\right)$. Let at least one of $E_{1}, E_{2}$ be stable. Then

$$
h^{0}\left(Q_{n}, E_{1}^{*} \otimes E_{2}\right)= \begin{cases}0 & \text { if and only if } E_{1}, E_{2} \text { are not isomorphic, } \\ 1 & \text { if and only if } E_{1}, E_{2} \text { are isomorphic. }\end{cases}
$$

PROOF. It is a straightforward extension of [OSS, corollary of Lemma 1.2.8 and Theorem 1.2.9, pp. 171-172]. 
THEOREM 2.8. (i) Let $n=2 m+1$ and let $S$ be the spinor bundle on $Q_{n}$. We have a natural exact sequence:

$$
0 \rightarrow S \rightarrow O_{Q_{n}}^{\oplus 2^{m+1}} \rightarrow S(1) \rightarrow 0
$$

and an isomorphism $S^{*} \simeq S(1)$.

(ii) Let $n=2 m$ and let $S^{\prime}, S^{\prime \prime}$ be the spinor bundles on $Q_{n}$. We have two natural exact sequences

$$
0 \rightarrow S^{\prime} \rightarrow \mathrm{O}_{Q_{n}}^{\oplus 2^{m}} \rightarrow S^{\prime \prime}(1) \rightarrow 0, \quad 0 \rightarrow S^{\prime \prime} \rightarrow \mathcal{O}_{Q_{n}}^{\oplus 2^{m}} \rightarrow S^{\prime}(1) \rightarrow 0 .
$$

Furthermore, if $n \equiv 0(\bmod 4)$ we have the two isomorphisms $S^{* *} \simeq S^{\prime}(1)$ and $S^{\prime \prime *} \simeq S^{\prime \prime}(1)$, and if $n \equiv 2(\bmod 4)$ we have the two isomorphisms $S^{\prime *} \simeq S^{\prime \prime}(1)$ and $S^{\prime \prime *} \simeq S^{\prime}(1)$.

PROOF. The proof is by induction on $n$. The result is well known for $n=2,3,4$. By definition of spinor bundles, if $S$ is a spinor bundle on $Q_{n}$ we have an exact sequence

$$
0 \rightarrow S \rightarrow \mathrm{O}^{\oplus 2^{[(n+1) / 2]}} \rightarrow E \rightarrow 0 .
$$

By the induction hypothesis (and Corollary 2.2) it follows that if $\left.E\right|_{Q_{n-1}}$ is semistable for the generic $Q_{n-1}$, then $E$ is semistable too.

Now let $n$ be odd. If we tensor (4) by $S$ and consider the associated cohomology sequence, we get by Theorem 2.3

$$
h^{0}(S \otimes E)=h^{1}(S \otimes S) .
$$

Let $Q_{n-1}$ be a smooth hyperplane section of $Q_{n}$. Look at the exact sequence

$$
\left.0 \rightarrow S \otimes S \rightarrow S \otimes S(1) \rightarrow S \otimes S(1)\right|_{Q_{n-1}} \rightarrow 0 .
$$

By the induction hypothesis, if $S^{\prime}$ and $S^{\prime \prime}$ are the spinor bundles on $Q_{n-1}$, we have from Theorem 1.4

$$
\left.S \otimes S(1)\right|_{Q_{n-1}} \simeq\left(S^{\prime} \otimes S^{\prime \prime *}\right)^{\oplus 2} \oplus\left(S^{\prime} \otimes S^{\prime *}\right) \oplus\left(S^{\prime \prime} \otimes S^{\prime *}\right) .
$$

Then by Lemma $2.7 h^{0}\left(\left.S \otimes S(1)\right|_{Q_{n-1}}\right)=2$.

From (5), we have an exact sequence

$$
H^{0}\left(Q_{n}, S \otimes S(1)\right) \rightarrow H^{0}\left(Q_{n-1},\left.S \otimes S(1)\right|_{Q_{n-1}}\right) \rightarrow H^{1}\left(Q_{n}, S \otimes S\right) .
$$

As $h^{0}(S \otimes S(1)) \leq 1$ by Lemma 2.7 , we have $h^{1}(S \otimes S) \neq 0$, and then $h^{0}(S \otimes E) \neq 0$. From Lemma 2.7 it follows that $E \simeq S^{*}$, and $h^{1}(S \otimes S)=1$. From (6) it follows that $h^{0}(S \otimes S(1)) \neq 0$, and from Lemma 2.7 again it follows that $S^{*} \simeq S(1)$.

Let $n$ be even. We have an exact sequence

$$
0 \rightarrow S^{\prime} \rightarrow \mathrm{O}^{\oplus 2^{n / 2}} \rightarrow E \rightarrow 0
$$

with $E$ semistable. If we tensor it by $S^{\prime \prime *}(-1)$ and we look at the associated cohomology sequence we get

$$
H^{0}\left(Q_{n}, S^{\prime \prime *}(-1) \otimes E\right) \rightarrow H^{1}\left(Q_{n}, S^{\prime} \otimes S^{\prime *}(-1)\right) \rightarrow 0 .
$$

Let $Q_{n-1}$ be a smooth hyperplane section of $Q_{n}$. Look at the exact sequence

$$
\left.0 \rightarrow S^{\prime} \otimes S^{\prime \prime *}(-1) \rightarrow S^{\prime} \otimes S^{\prime \prime *} \rightarrow S^{\prime} \otimes S^{\prime \prime *}\right|_{Q_{n-1}} \rightarrow 0
$$


By the induction hypothesis, if $S$ is the spinor bundle on $Q_{n-1}$, we have $\left.S^{\prime} \otimes S^{\prime \prime *}\right|_{Q_{n-1}} \simeq S \otimes S^{*}$, so that

$$
H^{0}\left(Q_{n-1},\left.S^{\prime} \otimes S^{\prime \prime *}\right|_{Q_{n-1}}\right)=\mathbb{C}
$$

From (9) we have an exact sequence

$$
\begin{gathered}
H^{0}\left(Q_{n}, S^{\prime} \otimes S^{\prime \prime *}\right) \rightarrow \mathbb{C} \rightarrow H^{1}\left(Q_{n}, S^{\prime} \otimes S^{\prime \prime *}(-1)\right) \\
\| \\
0
\end{gathered}
$$

so that $h^{1}\left(S^{\prime} \otimes S^{\prime \prime *}(-1)\right) \neq 0$. Then from (8) it follows that $H^{0}\left(S^{\prime \prime *}(-1) \otimes E\right) \neq 0$ and from Lemma $2.7 E \simeq S^{\prime \prime}(1)$.

If we tensor (7) by $S^{\prime}$ we get

$$
0 \rightarrow S^{\prime} \otimes S^{\prime} \rightarrow S^{\prime \oplus 2^{n / 2}} \rightarrow S^{\prime \prime}(1) \otimes S^{\prime} \rightarrow 0
$$

so that

$$
h^{0}\left(S^{\prime \prime}(1) \otimes S^{\prime}\right)=h^{1}\left(S^{\prime} \otimes S^{\prime}\right) .
$$

Look at the exact sequence

$$
\left.0 \rightarrow S^{\prime} \otimes S^{\prime} \rightarrow S^{\prime} \otimes S^{\prime}(1) \rightarrow S^{\prime} \otimes S^{\prime}(1)\right|_{Q_{n-1}} \rightarrow 0 .
$$

We have an associated cohomology sequence

$$
H^{0}\left(Q_{n}, S^{\prime} \otimes S^{\prime}(1)\right) \rightarrow H^{0}\left(Q_{n-1},\left.S^{\prime} \otimes S^{\prime}(1)\right|_{Q_{n-1}}\right) \rightarrow H^{1}\left(Q_{n}, S^{\prime} \otimes S^{\prime}\right) .
$$

Since, by the induction hypothesis, $H^{0}\left(Q_{n-1},\left.S^{\prime} \otimes S^{\prime}(1)\right|_{Q_{n-1}}\right)=\mathbb{C}$, from (11) and (13) we must have

$$
\begin{aligned}
\text { either } & h^{0}\left(S^{\prime} \otimes S^{\prime}(1)\right) \neq 0, \\
\text { or } & h^{0}\left(S^{\prime \prime}(1) \otimes S^{\prime}\right) \neq 0 .
\end{aligned}
$$

From Lemma 2.7 we must have respectively

$$
\begin{aligned}
\text { either } & S^{\prime *} \simeq S^{\prime}(1), \\
\text { or } & S^{\prime *} \simeq S^{\prime \prime}(1) .
\end{aligned}
$$

Now Theorem 2.6 gives the result.

REMARK 2.9. From Theorems $1.4,2.5,2.6$ and 2.8 it is possible to compute inductively the Chern classes of spinor bundles. (See [Fr] for a description of the intersection ring of $Q_{n}$.) With obvious notations, for $n \leq 8$ the Chern classes of spinor bundles on $Q_{n}$ are the following:

$$
\begin{aligned}
& n=2: c_{1}=(-1,0) \text { or }(0,-1)\left(\text { according to } S^{\prime} \text { or } S^{\prime \prime}\right), \\
& n=3: c_{1}=-1, c_{2}=1, \\
& n=4: c_{1}=-1, c_{2}=(1,0) \text { or }(0,1), \\
& n=5: c_{1}=-2, c_{2}=2, c_{3}=-2, c_{4}=0, \\
& n=6: c_{1}=-2, c_{2}=2, c_{3}=(-2,0) \text { or }(0,-2), c_{4}=0, \\
& n=7: c_{1}=-4, c_{2}=8, c_{3}=-10, c_{4}=16, c_{5}=-8, c_{6}=4, \\
& n=8: c_{1}=-4, c_{2}=8, c_{3}=-10, c_{4}=(11,5) \text { or }(5,11), c_{5}=-8, c_{6}=4, \\
& c_{7}=-2, c_{8}=1 .
\end{aligned}
$$

Obviously, on $Q_{n}(n \geq 3), c_{1}=-2^{[(n-3) / 2]}$. It seems hard to find simple general formulas for higher Chern classes.

The following theorem shows that spinor bundles are rigid. 
THEOREM 2.10. (i) Let $n$ be odd. If $S$ is the spinor bundle on $Q_{n}$, then $h^{1}\left(S \otimes S^{*}\right)=0$.

(ii) Let $n$ be even. If $S^{\prime}, S^{\prime \prime}$ are the spinor bundles on $Q_{n}$, then

$$
h^{1}\left(S^{\prime} \otimes S^{\prime *}\right)=h^{1}\left(S^{\prime} \otimes S^{\prime \prime *}\right)=h^{1}\left(S^{\prime \prime} \otimes S^{\prime \prime *}\right)=0 .
$$

PROOF. The proof is by induction on $n$. The case for $n=2$ is well known. If $n$ is odd, the cohomology sequence associated to (5) is

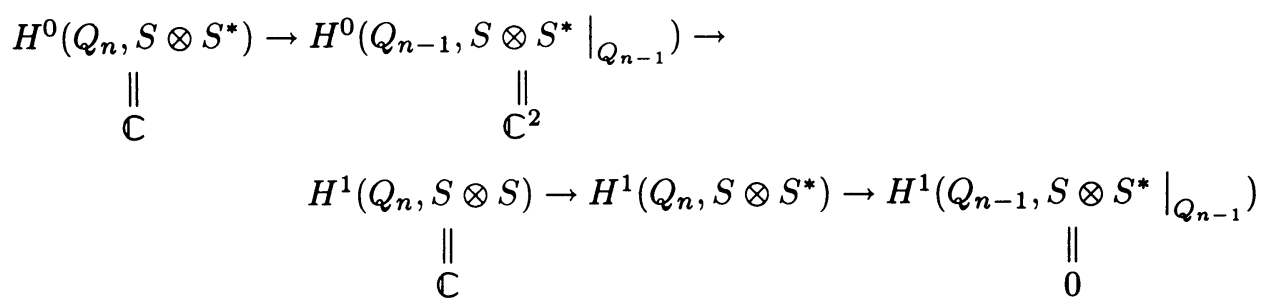

so that $h^{1}\left(S \otimes S^{*}\right)=0$ as we wanted.

If $n$ is even the proof is longer (according to $n \equiv 0,2(\bmod 4))$ though still elementary and will be omitted.

THEOREM 2.11. Let $E$ be a vector bundle on $Q_{n}$. Let $Q_{n-1}$ be a smooth hyperplane section of $Q_{n}$ (if $E$ is semistable we can omit the word "generic").

(i) Let $n$ be even and let $E$ be a vector bundle on $Q_{n}$ such that $\left.E\right|_{Q_{n-1}} \simeq S$ where $S$ is the spinor bundle an $Q_{n-1}$. Then $E$ is a spinor bundle on $Q_{n}$.

(ii) Let $n$ be odd and let $E$ be a vector bundle on $Q_{n}$ such that $\left.E\right|_{Q_{n-1}} \simeq S^{\prime} \oplus S^{\prime \prime}$, where $S^{\prime}, S^{\prime \prime}$ are the spinor bundles on $Q_{n-1}$. Then $E$ is the spinor bundle on $Q_{n}$.

Proof. First observe that by Corollary $2.2 E$ is semistable. From the cohomology sequence associated to the sequence

$$
\left.0 \rightarrow E(k-1) \rightarrow E(k) \rightarrow E(k)\right|_{Q_{n-1}} \rightarrow 0 \quad(k \in \mathbb{Z})
$$

we get that the map $H^{1}\left(Q_{n}, E(k-1)\right) \rightarrow H^{1}\left(Q_{n}, E(k)\right)$ is surjective $\forall k \in \mathbb{Z}$. From Theorem B and Serre duality it follows that

$$
H^{1}\left(Q_{n}, E(k)\right)=0 \quad \forall k \in \mathbb{Z} .
$$

Now let $n$ be even. Consider the sequence $\left(S^{\prime}, S^{\prime \prime}\right.$ spinor bundles on $\left.Q_{n}\right)$

$$
\left.0 \rightarrow E \otimes S^{\prime *}(-1) \rightarrow E \otimes S^{\prime *} \rightarrow E \otimes S^{\prime *}\right|_{Q_{n-1}} \rightarrow 0
$$

By hypothesis, $H^{0}\left(Q_{n-1},\left.E \otimes S^{\prime *}\right|_{Q_{n-1}}\right)=\mathbb{C}$, so that we have an exact sequence

$$
H^{0}\left(Q_{n}, E \otimes S^{\prime *}\right) \rightarrow \mathbb{C} \rightarrow H^{1}\left(Q_{n}, E \otimes S^{* *}(-1)\right) .
$$

It must be either $h^{0}\left(E \otimes S^{* *}\right) \neq 0$ or $h^{1}\left(E \otimes S^{* *}(-1)\right) \neq 0$. In the first case from Lemma 2.7 it follows that $E \simeq S^{\prime}$.

In the second case, by Theorem 2.8 we have the sequence

$$
0 \rightarrow E \otimes S^{\prime *}(-1) \rightarrow E^{\oplus 2^{n / 2}} \rightarrow E \otimes S^{\prime *} \rightarrow 0 .
$$

From the associated cohomology sequence we get

$$
H^{0}\left(Q_{n}, E \otimes S^{\prime *}\right) \rightarrow H^{1}\left(Q_{n}, E \otimes S^{\prime *}(-1)\right) \rightarrow 0
$$


so that $h^{0}\left(E \otimes S^{\prime * *}\right) \neq 0$. From Lemma 2.7 it follows that $E \simeq S^{\prime \prime}$ as we wanted.

If $n$ is odd, by the hypothesis, $h^{0}\left(\left.E \otimes S^{*}\right|_{Q_{n-1}}\right)=2$. Consider the sequence

$$
\left.0 \rightarrow E \otimes S^{*}(-1) \rightarrow E \otimes S^{*} \rightarrow E \otimes S^{*}\right|_{Q_{n-1}} \rightarrow 0
$$

From the associated cohomology sequence we get

$$
H^{0}\left(Q_{n}, E \otimes S^{*}\right) \rightarrow \mathbb{C}^{2} \rightarrow H^{1}\left(Q_{n}, E \otimes S^{*}(-1)\right) .
$$

By Lemma $2.7, h^{0}\left(E \otimes S^{*}\right) \leq 1$, so that $h^{1}\left(E \otimes S^{*}(-1)\right) \neq 0$.

By Theorem 2.8, we have the sequence

$$
0 \rightarrow E \otimes S^{*}(-1) \rightarrow E^{\oplus 2^{(n+1) / 2}} \rightarrow E \otimes S^{*} \rightarrow 0 .
$$

From the associated cohomology sequence we get

$$
H^{0}\left(Q_{n}, E \otimes S^{*}\right) \rightarrow H^{1}\left(Q_{n}, E \otimes S^{*}(-1)\right) \rightarrow 0
$$

so that $h^{0}\left(E \otimes S^{*}\right) \neq 0$, and by Lemma 2.5 it follows that $E \simeq S$.

3. Some moduli spaces of rank 3 vector bundles on $Q_{5}$ and $Q_{6}$.

REMARK 3.1. Observe that on $Q_{8}$, the duals of the spinor bundles $S^{\prime}, S^{\prime \prime}$ are globally generated vector bundles of rank 8 . As by Remark $2.9, c_{8}\left(S^{\prime *}\right)=c_{8}\left(S^{\prime \prime *}\right)=$ 1 , we get that the generic section of $S^{\prime *}$ (or $S^{\prime \prime *}$ ) vanishes only at a simple point.

In a similar way, observe that on $Q_{5}$ and $Q_{6}$ the duals of the spinor bundles (of rank 4) have their generic sections which do not vanish anywhere. Thus we can construct a trivial line subbundle of the dual of a spinor bundle $S$ on $Q_{5}$ (and $Q_{6}$ ), so that we have an exact sequence

$$
0 \rightarrow 0 \rightarrow S^{*} \rightarrow S^{*} / 0 \rightarrow 0
$$

where $S^{*} / 0$ is a rank 3 bundle.

We recall that on $Q_{4}$ Hernandez and Sols [HS] have constructed rank 3 bundles $E$ from the sequence $\left(S^{\prime}, S^{\prime \prime}\right.$ spinor bundles on $\left.Q_{4}\right)$

$$
0 \rightarrow 0 \rightarrow S^{\prime *} \oplus S^{\prime \prime *} \rightarrow E \rightarrow 0 .
$$

In [Ot1] this construction is extended to $\operatorname{Gr}(k, n)$.

From Theorem 1.4 we get the surprising consequence that the bundles $E$ in (15) extend to $Q_{5}$ and even to $Q_{6}$ to the bundles $S^{*} / 0$ in (14). Using the description of the moduli space given in [HS] we are able to prove the following:

THEOREM 3.2. (i) $\mathbb{P}^{7} \backslash Q_{6}$ is the fine moduli space of rank 3 stable vector bundles on $Q_{6}$ with Chern classes $c_{1}=2, c_{2}=2, c_{3}=(2,0)$ or $(0,2)$.

(ii) $\mathbb{P}^{7} \backslash Q_{6}$ is the fine moduli space of rank 3 stable vector bundles on $Q_{5}$ with Chern classes $c_{1}=2, c_{2}=2, c_{3}=2$.

PROOF. We want to show first that a rank 3 stable vector bundle $E$ on $Q_{6}$ with Chern classes $c_{1}=2, c_{2}=2, c_{3}=(2,0)$ arises as a quotient of the dual of the spinor bundle $S^{\prime *}$ (the one with $c_{3}\left(S^{\prime *}\right)=(2,0)$ ) in a sequence

$$
0 \rightarrow O \rightarrow S^{*} \rightarrow E \rightarrow 0
$$

Let $Q_{4} \subset Q_{5} \subset Q_{6}$ be smooth generic plane sections and $E$ a vector bundle as above. By a theorem of Maruyama [Ma2, Theorem 3.1] $\left.E\right|_{Q_{4}}$ is semistable, thus stable because

$$
\text { h.c.f. }\left(c_{1}(E), \operatorname{rank} E\right)=\text { h.c.f. }(2,3)=1 \text {. }
$$


By [HS], we have an exact sequence on $Q_{4}$

$$
\left.0 \rightarrow 0 \rightarrow \tilde{S}^{\prime} \oplus \tilde{S}^{\prime \prime} \rightarrow E\right|_{Q_{4}} \rightarrow 0
$$

where $\tilde{S}^{\prime}, \tilde{S}^{\prime \prime}$ are the spinor bundles on $Q_{4}$.

From the dual sequence of $(17)$ one easily computes $h^{2}\left(\left.E^{*}(t)\right|_{Q_{4}}\right)=0 \forall t \in \mathbb{Z}$, $h^{1}\left(\left.E^{*}(t)\right|_{Q_{4}}\right)=0$ for $t \leq-1, h^{1}\left(\left.E^{*}\right|_{Q_{4}}\right)=1$. Then, looking at the cohomology sequences associated to the sequences,

$$
\begin{aligned}
& \left.\left.\left.0 \rightarrow E^{*}(t-1)\right|_{Q_{5}} \rightarrow E^{*}(t)\right|_{Q_{5}} \rightarrow E^{*}(t)\right|_{Q_{4}} \rightarrow 0, \\
& \left.0 \rightarrow E^{*}(t-1) \rightarrow E^{*}(t) \rightarrow E^{*}(t)\right|_{Q_{5}} \rightarrow 0,
\end{aligned}
$$

we get successively (by Theorem B and Serre duality):

$$
\begin{aligned}
& h^{2}\left(\left.E^{*}(t)\right|_{Q_{5}}\right)=0 \quad \forall t \in \mathbb{Z}, \quad h^{1}\left(\left.E^{*}(t)\right|_{Q_{5}}\right)=0 \quad \text { for } t \leq-1, \quad h^{1}\left(\left.E^{*}\right|_{Q_{5}}\right)=1, \\
& h^{2}\left(E^{*}(t)\right)=0 \quad \forall t \in \mathbb{Z}, \quad h^{1}\left(E^{*}(t)\right)=0 \quad \text { for } t \leq-1, \quad h^{1}\left(E^{*}\right)=1 .
\end{aligned}
$$

In particular, the restriction maps

$$
H^{1}\left(Q_{5},\left.E^{*}\right|_{Q_{5}}\right) \rightarrow H^{1}\left(Q_{4},\left.E^{*}\right|_{Q_{4}}\right), \quad H^{1}\left(Q_{6}, E^{*}\right) \rightarrow H^{1}\left(Q_{5},\left.E^{*}\right|_{Q_{5}}\right)
$$

are both isomorphisms of one-dimensional vector spaces.

When we interpret $H^{1}\left(Q_{6}, E^{*}\right)$ in terms of extensions, we get that there is an exact sequence on $Q_{6}$,

$$
0 \rightarrow O \rightarrow A \rightarrow E \rightarrow 0
$$

which restricts on the generic $Q_{4}$ to (17), so that by Theorem $2.11 A \simeq S^{*}$ and (16) is proved (there is only one nonsplitting extension).

Next, observe that all bundles $E$ arising from (16) are stable, in fact one easily computes

$$
E_{\text {norm }}=E(-1), \quad\left(E^{*}\right)_{\text {norm }}=E^{*}, \quad h^{0}\left(E_{\text {norm }}\right)=h^{0}\left(\left(E^{*}\right)_{\text {norm }}\right)=0 .
$$

Furthermore, two bundles $E_{1}, E_{2}$ arising from (16) are isomorphic if and only if the two sections $s_{1}, s_{2}$ that define them are a scalar multiple one of the other. In fact each morphism $\varphi=S^{*} \rightarrow E$ can be uniquely extended to a morphism $\psi: S^{*} \rightarrow S^{*}$ by the vanishing of $h^{0}(S)$ and $h^{1}(S)$.

So we have a bijective map from a Zariski open set of $\mathbb{P}^{7}$ to the coarse moduli space $M$ (which exists by $[\mathbf{M a} \mathbf{1}])$.

The following lemma shows that $M$ is smooth, so that $M$ is exactly a Zariski open set of $\mathbb{P}^{7}$, and the description given shows that it is fine.

Precisely, we have $M=\mathbb{P}^{7} \backslash Q_{6}$. In order to prove this claim, consider that by the triality principle [Ti, §3.1] the image of the embedding $Q_{6} \rightarrow \operatorname{Gr}(3,7)$ which defines the spinor bundles can be described as the set of linear $\mathbf{P}^{3}$ in $\mathbf{P}^{7}$ lying in some quadric $Q_{6} \subset \mathbb{P}^{7}$. The cycles $\left\{\mathbf{P}^{3} \mid \mathbf{P}^{3} \subset \mathbf{P}^{6}\right.$ with $\mathbf{P}^{6}$ fixed hyperplane $\}$ are the zero loci of sections of the dual of the universal bundle of $\operatorname{Gr}(3,7)$. There exists a linear $\mathbf{P}^{3} \subset Q_{6}$ lying in a hyperplane if and only if the hyperplane is tangent to $Q_{6}$.

Then the sections of $S^{*}$ corresponding to the hyperplanes tangent to $Q_{6}$ are the only one for which the quotient $S^{*} / 0$ is not locally free. These sections vanish on a linear $\mathrm{P}^{3}$ contained in $Q_{6}$. This completes the proof of (i). Observe now that the 
embedding $Q_{5} \rightarrow \operatorname{Gr}(3,7)$ which defines the spinor bundle on $Q_{5}$ factors through $Q_{6}$. It follows that the proof of (ii) is very similar to the proof of (i). We omit the details.

LEMMA 3.3. Let $S$ be a spinor bundle on $Q_{6}$. Let $E$ be a vector bundle on $Q_{6}$ arising from the sequence

$$
0 \rightarrow O \rightarrow S^{*} \rightarrow E \rightarrow 0
$$

Then $h^{1}\left(E \otimes E^{*}\right)=7$.

PROOF. Tensoring by $S^{*}$ the dual sequence of (16), we get

$$
0 \rightarrow E^{*} \otimes S^{*} \rightarrow S \otimes S^{*} \rightarrow S^{*} \rightarrow 0 \text {. }
$$

From the associated cohomology sequence and Theorem 2.10 we have

$$
0 \rightarrow H^{0}\left(Q_{6}, E^{*} \otimes S^{*}\right) \rightarrow H^{0}\left(Q_{6}, S \otimes S^{*}\right) \rightarrow H^{0}\left(Q_{6}, S^{*}\right) \rightarrow H^{1}\left(Q_{6}, E^{*} \otimes S^{*}\right) \rightarrow 0
$$

so that $h^{0}\left(E^{*} \otimes S^{*}\right)-h^{1}\left(E^{*} \otimes S^{*}\right)=-7$.

From the cohomology sequence associated to the dual sequence of (16) we get $h^{0}\left(E^{*}\right)=0, h^{1}\left(E^{*}\right)=1, h^{2}\left(E^{*}\right)=0$. Then, after tensoring $(16)$ by $E^{*}$ and looking at cohomology we have

$$
\begin{aligned}
& 0 \rightarrow H^{0}\left(Q_{6}, S^{*} \otimes E^{*}\right) \rightarrow H^{0}\left(Q_{6}, E \otimes E^{*}\right) \rightarrow H^{1}\left(Q_{6}, E^{*}\right) \rightarrow H^{1}\left(Q_{6}, S^{*} \otimes E^{*}\right) \\
& \|\quad\| \\
& \text { C } \\
& \text { C } \\
& \rightarrow H^{1}\left(Q_{6}, E \otimes E^{*}\right) \rightarrow 0
\end{aligned}
$$

so that $h^{1}\left(E \otimes E^{*}\right)=h^{1}\left(E^{*} \otimes S^{*}\right)-h^{0}\left(E^{*} \otimes S^{*}\right)=7$, as we wanted.

REMARK 3.4. We do not know if a semistable vector bundle on $Q_{n}$ with the same rank and Chern classes of a spinor bundle is a spinor bundle. For $n \leq 4$ this is true (using the same techniques of [HS]).

In $\S 1$ we noted that the variety $S_{3}$ which parametrizes one family of the linear $\mathbb{P}^{3}$ 's contained in $Q_{6}$ is isomorphic to the variety which parametrizes the linear $\mathbf{P}^{2}$ 's contained in $Q_{5}$. By the triality principle, $S_{3} \simeq Q_{6}$ [Ti]. So, with obvious notations, we have the isomorphisms (recall that $G_{n}=\operatorname{Spin}(n+2)$ ): $Q_{6} \simeq G_{6} / P\left(\alpha_{1}\right) \simeq$ $G_{6} / P\left(\alpha_{3}\right) \simeq G_{6} / P\left(\alpha_{4}\right) \simeq G_{5} / P\left(\alpha_{3}^{\prime}\right), Q_{5} \simeq G_{5} / P\left(\alpha_{1}^{\prime}\right)$.

THEOREM 3.5. A rank 3 stable vector bundle on $Q_{6} \simeq \operatorname{Spin}(7) / P\left(\alpha_{3}^{\prime}\right)$ with Chern classes $c_{1}=2, c_{2}=2, c_{3}=(2,0)$ or $(0,2)$ is defined by the irreducible representation of the semisimiple part of $P\left(\alpha_{3}^{\prime}\right)$ with maximal weight $\lambda_{1}$.

PROOF. Consider the following three diagrams given by incidence relations:

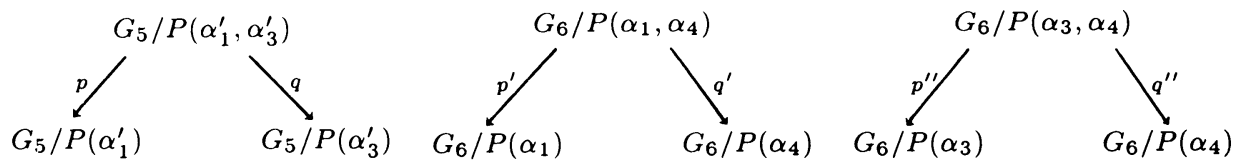


The dual of the two spinor bundles on $Q_{6}$ are defined by $S^{\prime *}=q_{*}^{\prime} p^{\prime *} O(1)$, $S^{\prime \prime *}=q_{*}^{\prime \prime} p^{\prime \prime *} O(1)$. So in this case the spinor bundles are the pullback of the universal bundles by the two embeddings $Q_{6} \simeq S_{3} \rightarrow \operatorname{Gr}(3,7)$ corresponding to the two families of linear $\mathbb{P}^{3}$ 's contained in $Q_{6}$.

In the same way the 3-bundle $q_{*} p^{*} O(1)=U^{*}$ on $Q_{6}=G_{5} / P\left(\alpha_{3}^{\prime}\right)$ is the pullback of the dual of the universal bundle by the embedding $Q_{6} \simeq G_{5} / P\left(\alpha_{3}^{\prime}\right) \rightarrow \operatorname{Gr}(2,6)$ corresponding to the family of linear $\mathbb{P}^{2}$ 's contained in $Q_{5} . U^{*}$ is defined by the irreducible representation of the semisimple part of $P\left(\alpha_{3}^{\prime}\right)$ with maximal weight $\lambda_{1}$.

We have two exact sequences of vector bundles on $Q_{6}$ :

$$
0 \rightarrow U \rightarrow S^{\prime} \rightarrow \mathrm{O} \rightarrow 0, \quad 0 \rightarrow U \rightarrow S^{\prime \prime} \rightarrow 0 \rightarrow 0 .
$$

Now the thesis follows from Theorem 3.2 dualizing the sequences (18).

THEOREM 3.6. (i) Let $E$ be a stable 3-bundle on $Q_{6}$ with Chern classes $c_{1}=2$, $c_{2}=2, c_{3}=(2,0)$ or $(0,2)$. Then

$$
\frac{1}{120}(t+1)(t+2)(t+3)(t+4)(t+5)(t+7)= \begin{cases}h^{0}\left(Q_{6}, E(t)\right) & \text { for } t \geq 0, \\ h^{6}\left(Q_{6}, E(t)\right) & \text { for } t \leq-8 .\end{cases}
$$

All other values of $h^{i}\left(Q_{6}, E(t)\right)$ vanish except $h^{5}\left(Q_{6}, E(-6)\right)=1$.

(ii) Let $E$ be a stable 3-bundle on $Q_{5}$ with Chern classes $c_{1}=2, c_{2}=2, c_{3}=2$. Then

$$
\frac{1}{120}(t+1)(t+2)(t+3)(t+4)(6 t+35)= \begin{cases}h^{0}\left(Q_{5}, E(t)\right) & \text { for } t \geq 0, \\ -h^{5}\left(Q_{5}, E(t)\right) & \text { for } t \leq-6 .\end{cases}
$$

All other values of $h^{i}\left(Q_{5}, E(t)\right)$ vanish except $h^{4}\left(Q_{5}, E(-5)\right)=1$.

(iii) Let $E$ be a stable 3 -bundle on $Q_{4}$ with Chern classes $c_{1}=2, c_{2}=(2,2)$, $c_{3}=2$. Then

$$
\frac{1}{12}(t+1)(t+2)(t+3)(3 t+14)= \begin{cases}h^{0}\left(Q_{4}, E(t)\right) & \text { for } t \geq 0 \\ h^{4}\left(Q_{4}, E(t)\right) & \text { for } t \leq-5 .\end{cases}
$$

All other values of $h^{i}\left(Q_{4}, E(t)\right)$ vanish except $h^{3}\left(Q_{4}, E(-4)\right)=1$.

PROOF. On $Q_{6}$ the result follows after some computations from Theorem 3.5 and the theorem of Bott. On $Q_{5}$ it follows by restricting the bundles from $Q_{6}$ to a smooth hyperplane section and by the cohomology sequence associated to the exact sequence on $Q_{6}$,

$$
\left.0 \rightarrow E(t-1) \rightarrow E(t) \rightarrow E(t)\right|_{Q_{5}} \rightarrow 0 .
$$

In the same way we get the result on $Q_{4}$.

\section{REFERENCES}

[At] M. Atiyah, On the Krull-Schmidt theorem with application to sheaves, Bull. Soc. Math. France 84 (1956), 306-317.

[Ba] E. Ballico, Uniform vector bundles on quadrics, Ann. Univ. Ferrara VII 27 (1981), 135-146.

[Be] A. A. Beilinson, Coherent sheaves on $\mathbf{P}^{n}$ and problems of linear algebra, Funktsional. Anal. i Prilozhen. 12 (1978), 68-69; English transl., Functional Anal. Appl. 12 (1978), 214-216.

[Fr] K. Fritzsche, Linear-Uniforme Bundel auf Quadriken, Ann. Sci. Norm. Sup. Pisa (4) 10 (1983), 313-339.

[GH] P. Griffiths and J. Harris, Principles of algebraic geometry, Wiley, New York, 1978. 
[HS] R. Hernandez and I. Sols, On a family of rank 3 bundles on $\mathrm{Gr}(1,3)$, J. Reine Angew. Math. 360 (1985), 124-135.

[LV] R. Lazarsfeld and A. Van de Ven, Topics in the geometry of projective spaces, Recent work of F. L. Zak, DMV Seminar 4, Birkhäuser, 1984.

[Ma1] M. Maruyama, Moduli of stable sheaves. I; II, J. Math. Kyoto Univ. 17 (1977), 91-126; 18 (1978), 557-614.

[Ma2] _ Boundedness of semistable sheaves of small ranks, Nagoya Math. J. 78 (1980), 65-94.

[OSS] C. Okonek, M. Schneider and M. Spindler, Vector bundles on complex projective spaces, Birkhauser, 1980.

[Ot1] G. Ottaviani, A class of n-bundles on $\operatorname{Gr}(k, n)$, J. Reine Angew. Math. 379 (1987), $182-208$.

[Ot2] _ Some extensions of Horrocks criterion to vector bundles on Grassmannians and quadrics, preprint.

[St] M. Steinsieck, Uber homogenen-rationale Mannigfaltigkeiten, Schriftenr. Math. Inst. Univ. Münster (2) 23 (1982), 1-55.

[Ti] J. Tits, Sur la trialité et certains groupes qui s'en deduisent, Inst. Hautes Études Sci. Publ. Math. 2 (1959), 13-60.

[Um] H. Umemura, On a theorem of Ramanan, Nagoya Math. J. 69 (1978), 131-138.

Istituto Matematico, Ulisse Dini, Viale Morgagni 67/A, 50134 Firenze, Italy 